\title{
What Motion Patterns Tell Us about Soccer Teams
}

\author{
Jörn Sprado and Björn Gottfried \\ Centre for Computing Technologies, University of Bremen, Germany \\ \{sprado,bg\}@tzi.de
}

\begin{abstract}
A qualitative representation of motion patterns is presented that forms an interface between low-level concepts of behaviours and high-level concepts of reasoning. How the patterns can be employed for characterising interaction patterns in soccer is demonstrated using the simulation league; also, specific soccer scenes from real games prove their adequacy. The advantages of our approach are: it supports the limited abilities of robots in the different RoboCup leagues, i. e. it relies on coarse positional distinctions that are reliably obtainable and easily translated into action; the analysis is directly derived from raw data without the need for any preprocessing steps; both situations can be dealt with, egocentric viewpoints of individuals and the bird's eye view; the approach is independent on the domain, i. e. generalises to arbitrary spatiotemporal interaction patterns.
\end{abstract}

\section{Introduction}

This paper investigates motion patterns among soccer players for analysing team behaviours. The purpose of this analysis is to find patterns which are typical for a specific team [714, for their defenders or other subgroups, and to come to specific conclusions about successful and unsuccessful behaviours in order to find promising team interactions. More advanced topics include the automatic search for specific situations in databases of recorded games up to the automatic recognition or even realisation of strategies.

A more specific sub-goal we shall focus on consists in characterising adequately specific scenes. The adequacy derives from a description which considers the specific role of each player, namely his viewpoint which determines how he interacts with others. This egocentric viewpoint has to rely on as simple distinctions as whether another player is left of him, in front of him, towards his right, or back. More precise descriptions would not reflect the distinctions a player, or rather a robot, is capable to make reliably. It is therefore the question, how a behaviour model looks like that captures those distinctions and whether such a model sufficiently characterises scenes of soccer games.

The model of motion patterns we shall finally arrive at has the following characteristics:

(a) It forms an interface between low-level concepts of behaviours and high-level concepts of reasoning.

L. Iocchi et al. (Eds.): RoboCup 2008, LNAI 5399, pp. 614625. 2009.

(C) Springer-Verlag Berlin Heidelberg 2009 
(b) Giving the limited abilities of robots in the different RoboCup leagues, the proposed coarse qualitative motion patterns are well suited for they do only require simple abilities regarding both sensors and effectors; the patterns rely on coarse positional distinctions that are reliably obtainable and that can be easily translated into action.

(c) The qualitative behaviour patterns can be directly derived without any preprocessing steps, making the algorithms defined on them particularly fast and applicable for realtime analysis (cf. [1]).

(d) The proposed motion pattern representation is suited for both cases to deal with the egocentric viewpoint of individuals and for analysing a scene from the bird's eye view.

(e) The approach generalises to arbitrary spatiotemporal behaviour investigations that can built upon coarse qualitative patterns.

This approach is employed for characterising behaviour patterns in soccer using the complete 2d-simulation league championships of 2007; in addition, specific soccer scenes from real games prove the adequacy of this approach.

\subsection{Overview}

After having stated fundamental problems that characterise more thoroughly the situation, the most similar related work on motion patterns is reviewed. A number of basic motion patterns are introduced afterwards. These patterns are used in the following in order to analyse how they behave when considering different temporal and spatial granularity levels; this is for the purpose of showing that the approach handles raw positional data without any preprocessing steps. A number of combined patterns are introduced in the next section, showing how simple patterns combine to more sophisticated interaction behaviours. Such interaction behaviours are employed afterwards, giving first insights into how the approach will manage more sophisticated issues in future work, for example the description and recognition of tactics.

\section{Motion Analysis}

The spatiotemporal behaviour of objects can be analysed in many different ways. The obvious thing to do would be to take the positions of all objects for all time points in every temporal window of interest. Then however many problems arise:

- Which temporal windows are of interest?

- What is an appropriate temporal granularity level for the time points?

- What do we have to do with those many positions of each object in each time window in order to obtain meaningful patterns?

- How to integrate the great number of positions of different objects in different time windows in order to obtain information about how objects interact? 


\subsection{Related Methods}

Each method for the analysis of spatiotemporal behaviours has to deal with these problems. In particular the need to represent interactions is of great significance in order to evaluate team behaviour. However many of the methods restrict themselves to single trajectories: 12 consider only single trajectories instead of object interactions; [6] relate trajectories to the spatial context of a soccer pitch but they do also not consider object interactions; 9] provide a framework for intention recognition based on motion patterns, but again, do not consider interactions.

From those methods that have been applied in the soccer context 10 also considers interactions. They compare motion attributes of objects such as speed, change in speed, and motion azimuth; patterns are matched on these attributes in order to describe constancy (sequences of equal attributes for consecutive time steps), concurrence (objects showing the same attributes), and change (the change of attributes). Based on these patterns more complex patterns can be defined, such as trend-setter: an object anticipating motion attributes of other objects. This approach as well as others using such fundamental attributes have the disadvantage that there is the need to define interactions based on basic motion attributes before comprehensible patterns can be derived. An approach capturing directly significant object interactions would represent in a compact way complex behaviours of a group of objects.

Another approach applied to the soccer simulation league considers similar attributes such as motion azimuth, distances, and their changes for deriving also information about speed [11. They calculate four types of time series from the raw positional information of soccer players: the motion direction and speed of individual players, as well as the directions and distances among pairs of objects. Then two steps of abstraction are performed, namely the temporal segmentation of the time series into time intervals of homogeneous motion and a mapping of the attribute values describing the intervals to qualitative classes; the latter being domain dependent concepts which can be further processed by a rule-based interpretation system based on predicate logic.

\section{Basic Patterns}

Looking for an appropriate repertoire of motion patterns for modelling interactions the relationships between objects should be directly taken into account. These relationships should be defined in a relative way in order to directly capture relative object motions which do not require to take into account absolute
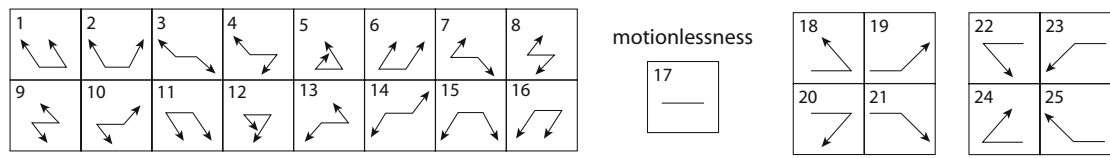

Fig. 1. Atomic motion patterns: $16 \mathcal{T} \mathcal{L} \mathcal{T}$ and $4 \mathcal{B L} \mathcal{T}$ classes 


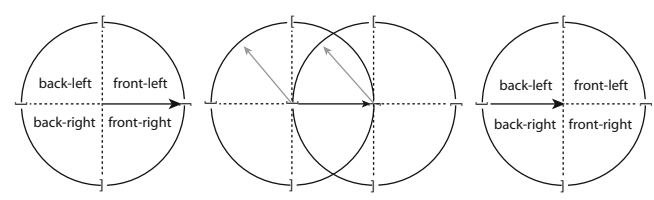

Fig. 2. How $\mathcal{T} \mathcal{L} \mathcal{T}(1)$ is defined

positions. In this way we model object interactions as defined in [5] which are shown in Fig. 1) since each pattern describes object interactions by using three line segments these patterns are referred to as Tripartite Line Tracks, $\mathcal{T} \mathcal{L} \mathcal{T}$ for short. In each pattern two objects as well as their positional relation among two time points is described; the middle line segment connects the positions of both objects at $t_{0}$ while the heads of the arrows show their positions at $t_{1}$. In this way, each $\mathcal{T} \mathcal{L} \mathcal{T}$ shows the spatial interaction between two objects, implying the consideration of relative positions and directions of movement.These patterns satisfy two important conditions: they can be generated efficiently and they can be easily comprehended; this enables their application in the context of game analysis. Even more important is that the patterns take into account the viewpoints of individual players: for this purpose the orientation grid of [15] is employed, there, motivated from the cognitive point of view. Fig. 2 shows how the orientation grid defines these patterns by taking the first pattern, $\mathcal{T} \mathcal{L} \mathcal{T}(1)$, as an example; it also shows its adequacy for the egocentric point of view, because it is simple to reliably make the distinctions between left, right, front, and back of one's own perspective; it is however also interesting for the coach in that the very same distinctions can be easily made from the bird's eye view.

While the motion patterns of the previous paragraph describe how two players move relative to each other between two time points, it might occur that one of the players does not move or that even both keep where they are. While the latter is the trivial pattern that connects two positions (referred to as motionlessness), in the former case four possibilities (referred to as semi-motionlessness) exist for each of the two players which are shown on the right hand side of Fig. 1. As a consequence there is a total of $16+(2 \cdot 4)+1=25$ basic motion interactions we shall distinguish.

\section{Temporal and Spatial Granules}

The aim is to provide efficient methods for game analysis, i. e. methods that directly process the raw data of tracked objects. That is, instead of accumulating time series [1] which are to be segmented into meaningful intervals, which in turn are to be categorised with respect to domain knowledge, it would be of great advantage to avoid the segmentation step. This is mainly because both threshold based and monotonicity based criteria for the segmentation of time series (both applied in [1]) are problematic since they apply general criteria to specific scenes that might significantly vary. A method that directly processes the raw positional 
data would be of advantage, since it avoids the representational vagueness of the segmentation process. To test such a method it is necessary to analyse the raw positional data of tracked objects regarding our proposed representation of interaction patterns. While all real world leagues require to track all objects of interest, in the case of the $2 \mathrm{~d}$-simulation league positions are directly given (by server logfiles). For a comprehensive investigation we have considered all 88 games of the RoboCup Championships 2007 in Atlanta. The results of the analysis have to reveal whether the raw positional data are in fact appropriate for game analysis taking into account the temporal and spatial dimensions. First hints would be that the motion pattern distributions do not significantly vary among different games which are analysed on the same temporal and spatial granularity levels. After that, for single specific scenes it is also to be shown that typical behaviour patterns are adequately characterised by our approach.

\subsection{Temporal Granularity}

The temporal granularity concerns the temporal distance between two successive states of a game. In a sense, for each real word league the temporal granularity is the same and from the point of view of the human observer continuous spatiotemporal patterns arise. For an appropriate analysis however the question arises as to how large the temporal distance between successive states should be. Regarding the simulation leagues the temporal dimension is partitioned into discrete slices: a number of 6000 snapshots define a game. These temporal slices can be directly taken for the analysis of such simulation games and for real word leagues similar slices are to be explicitly defined.

The first analysis looks for differences occurring when changing the temporal granularity. This analysis is of importance since an appropriate temporal granularity level is to be chosen for all other investigations. The result of this analysis shows that the number of $\mathcal{T} \mathcal{L} \mathcal{T}(1), \mathcal{T} \mathcal{L} \mathcal{T}(6), \mathcal{T} \mathcal{L}(11)$, and $\mathcal{T} \mathcal{L} \mathcal{T}(16)$ patterns increase when choosing coarser granules, while the number of those patterns decreases for which one or both objects do not change their positions. An explanation is that at finer temporal granules it occurs more often that players roughly keep where they are, while at coarser temporal granules the distance among successive positions increases. Of great interest is the dominance of $\mathcal{T} \mathcal{L} \mathcal{T}(1), \mathcal{T} \mathcal{L} \mathcal{T}(6), \mathcal{T} \mathcal{L} \mathcal{T}(11)$, and $\mathcal{T} \mathcal{L} \mathcal{T}(16)$ patterns at each temporal granularity level. It shows that objects almost ever move into similar directions, namely towards that direction where the main action of a game takes place; this is a clear tendency shown in most games.

The temporal granularity has been analysed by using a spatial granularity (see below) of 0.1 . For the finest case, i. e. a granularity of 1 (each time point is taken into account) the standard deviation is 0.79 , for a temporal granularity of 500 the standard deviation is 0.93 ; it shows that on each of the temporal granularity levels the games show similar motion pattern distributions. In other words the frequency distributions are very similar for all of the 88 games, even on quite coarse granularity levels. At a temporal granularity level of 500 however significant distinctions disappear, as shown by the black bars and the patterns 


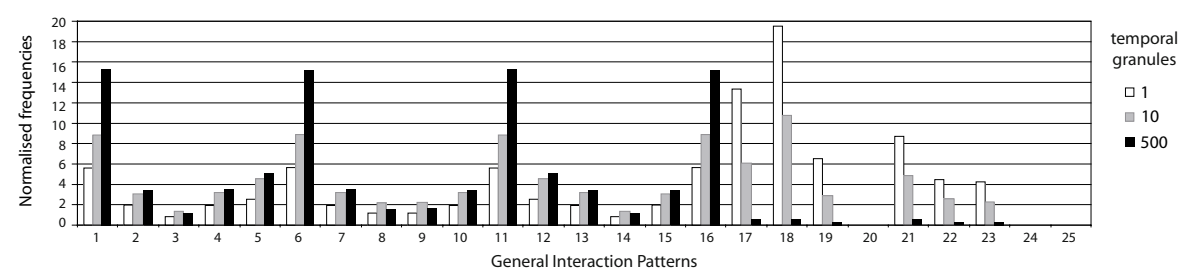

Fig. 3. Different temporal granules compared

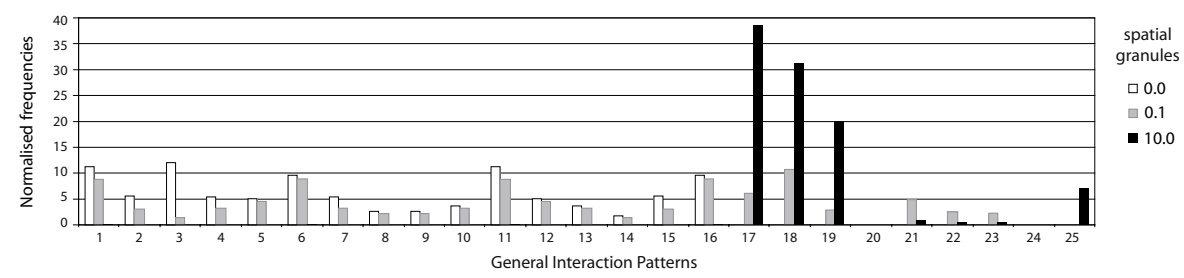

Fig. 4. Different spatial granules compared

on the right hand side of Fig. 3, i. e. patterns 17 to 25. Therefore, a granularity level should be chosen at which these patterns can be still distinguished; this is still the case at a temporal granularity level of 10 . Note that at this granularity level we not only work on the raw positional data but even have to take into account only a tenth part of the original data.

\subsection{Spatial Granularity}

The positional data of the $2 \mathrm{~d}$-simulation league is very precise; small changes are not visible. As a consequence a parameter is used that can be adjusted to an appropriate spatial granularity level, that is a level at which changes in position are visible. This brings in the problem that many small successive positional changes would be regarded as positional idleness, although they together sum up to large positional changes. We treat this problem by choosing a temporal granularity level, $t$, that correlates with the spatial granularity level, $s$, as follows: the larger the spatial threshold, the more positional changes will be regarded as spatial idleness; the temporal threshold has to be accordingly high: $t=f(s)$; the function $f$ assures that $\mathrm{t}$ is large enough in comparison to the spatial granularity. Conversely, the lower $s$, the finer the temporal granules can be chosen.

Fig. 4 shows that when the spatial granularity level is too large then the obtained motion patterns are quite inaccurate, for there are many idle motion patterns (mainly patterns 17, 18, and 19); on the other hand the interesting patterns between 1 and 16 disappear. The experiments indicate that a spatial granularity of 0.1 (in terms of the spatial unit of the 2d-simulation league) is appropriate together with a temporal granularity level of 10 (cycles), since at 


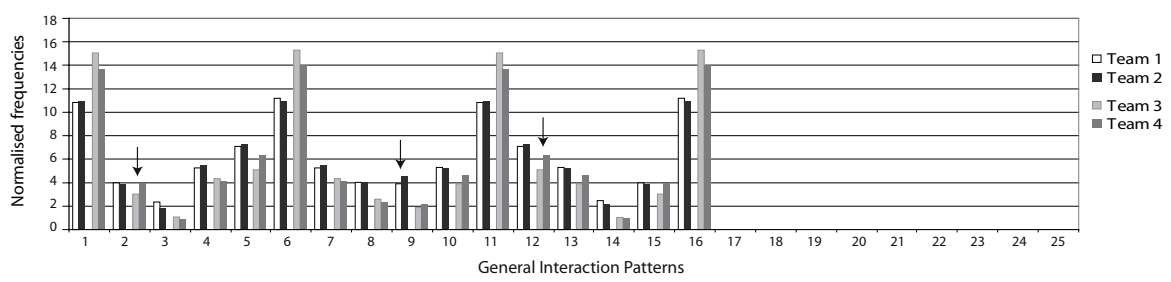

Fig. 5. Two games with four different teams

this granularity levels there is no significant loss in information compared with the raw data.

\subsection{Reactivity Behaviour}

Single games have been analysed as follows: the motion patterns between each pair of teammates have been determined; on the other hand, patterns among two players of different teams have not been considered. The purpose of this restriction is to measure the behaviour of teams as opposed to how a single player behaves in relation to all other opponents. Fig. 5 shows two games (the first one of loser round 1 , the second is the final game), i. e. for altogether four different teams the frequency distributions of interactions are shown. Team 1 (AT Humboldt) played against team 2 (NCL), and team 3 (Brainstormers) against team 4 (WrightEagle). Clearly, in both games the frequency distributions are very similar. There are only a few outliers, such as those three indicated by arrows between team 1 and team 2. An explanation for the similarity of the frequency distribution of opposing teams is that the team behaviours are quite reactive (cf. [13]), entailing similar behaviours. This can in fact be comprehended very well when observing games in the simulation league. Note that this observation is not specific to these two games but shown in most of the 88 games of the 2007 championships. Furthermore, we have chosen deliberately one game of the loser round 1 and the final game; in other words this behaviour is shown in very different games. We assume that in human soccer such reactivity behaviours will not be as dominant as in simulation soccer.

Comparing two different games it shows however that the frequency distributions differ (Fig. 5), i. e. the frequencies of team 1 and team 2 are clearly different to those of team 3 and team 4 . This simply indicates differences concerning the dominance of interaction patterns in different games.

\section{Specific Scenes}

Having analysed all 88 games from a holistic point of view, i. e. by determining histograms of motion patterns for the entire games, we will now turn our attention to specific scenes, in order to investigate whether the motion patterns are also appropriate for characterising specific interaction patterns in a game. 
A specific scene is characterised by a number of motion patterns which define how the players behave in that scene. Several characteristic behaviour patterns are analysed in the following paragraphs. That is, basic patterns can be combined to arbitrarily complex patterns in order to model interaction patterns of a number of $k>2$ players and along the temporal dimension.

Definition 1 (Motion Pattern). A motion pattern is a 5 -tuple $\left(m, o_{1}, o_{2}, t_{0}, t_{1}\right)$ with two objects $o_{1}, o_{2} \in \mathcal{O}$ among which a motion pattern $m \in \mathcal{M}$ holds between two time point $t_{0}, t_{1} \in \mathbb{N}$ with $t_{0}<t_{1}$.

Definition 2 (Complex Motion Pattern). A complex motion pattern is a conjunctive set of motion patterns.

\subsection{Complex Temporal Patterns}

This category of patterns combines $\mathcal{T} \mathcal{L} \mathcal{T}$ s along the temporal dimension: for two successive time intervals the basic patterns are identified, defining a more complex pattern.

Definition 3 (Complex Temporal Motion Pattern). A complex temporal motion pattern is a conjunctive set of motion patterns for successive time intervals.

Fig. 6] illustrates a complex temporal pattern. The meaning of such a passing manoeuvre depends on whether both players are teammates or not. In addition, the direction of such a passing manoeuvre regarding the pitch is of significance; this direction can be determined by defining motion patterns between players and specific landmarks, e. g. the corners of the pitch.

\subsection{Complex Spatial Patterns}

Fig. [7 shows a complex spatial pattern in the 73rd minute of the game GermanyCosta Rica of the world championship 2006: three players of the white team (Costa Rica) attack the black team (Germany); player 1 (left most) tries to run towards the front middle in order to receive the ball the attacker is going to pass through the German players. Table 1 shows the motion patterns for the scenario shown in Fig. 7 (Scene 1) and Fig. 9 (Scene 2). The motion patterns have been determined among all players within each team and among players of different teams.

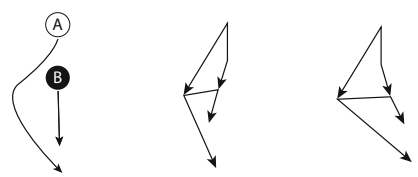

Fig. 6. Complex temporal patterns: passing manoeuvre with different meanings depending on whether both players are teammates or not; differences occur since B might turn to the left, $\mathcal{T} \mathcal{L} \mathcal{T}(11) \mathcal{T} \mathcal{L} \mathcal{T}(12)$, or to the right, $\mathcal{T} \mathcal{L} \mathcal{T}(10) \mathcal{T} \mathcal{L}(11)$ 

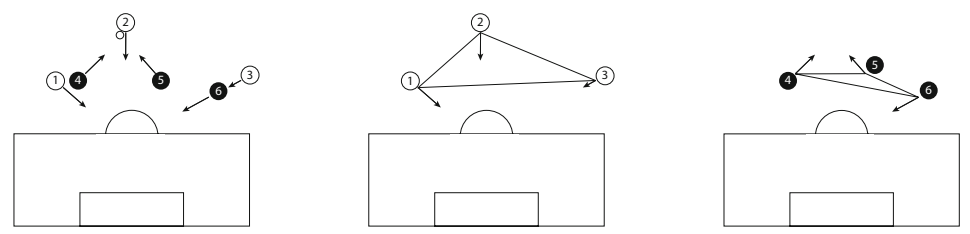

Fig. 7. White attacks black (left); motion patterns among teammates (middle \& right)
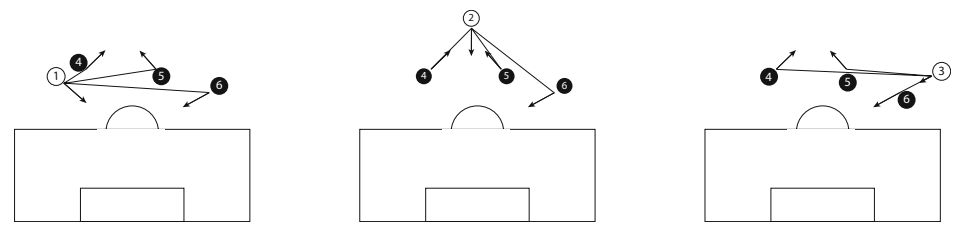

Fig. 8. Motion patterns among opponents

Table 1. $\mathcal{T} \mathcal{L} \mathcal{T}$ motion patterns for two scenes

\begin{tabular}{llll}
\hline & White Team & Black Team & Both Teams \\
\hline Scene 1 & $12,12,12$ & $05,08,04$ & 10, 09, 12, 05, 12, 12, 08, 07, 06 \\
Scene 2 & $04,04,12$ & 05, 08, 04 & 02, 01, 04, 05, 12, 12, 08, 07, 06 \\
\hline
\end{tabular}
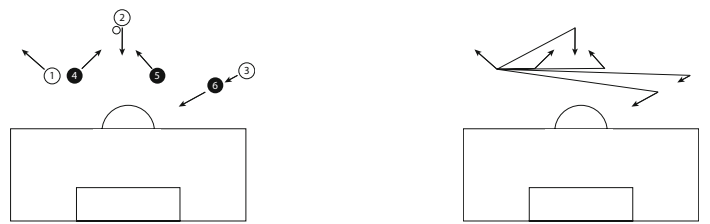

Fig. 9. Scene 2: no. 1 moves towards another direction, entailing the change of patterns

Definition 4 (Complex Spatial Motion Pattern). A complex spatial motion pattern is a conjunctive set of motion patterns of a number of $k>2$ objects.

The pattern among the teammates of Costa Rica (middle of Fig. 17) is an attack pattern in which all players move towards the goal. No. 1 runs in the penalty area, preparing for getting the pass from no. 2. The pattern among the German players (right of Fig. 7) is a typical defense pattern: two of the teammates show the tendency to meet in order to avoid a gap and to get the ball. That is Metzelder (no. 4) and Mertesacker (no. 5) move towards the attacker while Friedrich (no. 6) moves towards the middle expecting the ball in the middle, trying to beat Wanchope. Comparing these two patterns within the teams it clearly shows that the strategies of individual players (of the Costa Rica team) and their relations to their teammates follow one strategy, while the German defenders follow simultaneously two different strategies: no. 4 and no. 5 fight the 
attacker, while no. 6 prepares for dealing with no. 1 of the other team. A common strategy of the German team could have been an offside trap. For this purpose no. 6 would have had to avoid to run towards the goal too deeply. This alternative strategy can be easily communicated to human players. But even formally such an alternative strategy can be characterised using $\mathcal{T} \mathcal{L} \mathcal{T}$ motion patterns: no. 6 would have to avoid a motion pattern like $\mathcal{T} \mathcal{L} \mathcal{T}(12)$ with no. 1 as shown on the left hand side of Fig. 8 and should rather try to realise a pattern like $\mathcal{T} \mathcal{L} \mathcal{T}(9)$. Clearly, a finer argumentation would be that no. 6 could maintain pattern $\mathcal{T} \mathcal{L} \mathcal{T}(12)$, however, by simultaneously running not closer to the goal than no. 1. This strategy is much more difficult to realise for a robot with limited running abilities and sensors. By contrast, the realisation of $\mathcal{T} \mathcal{L} \mathcal{T}(9)$ instead of $\mathcal{T} \mathcal{L} \mathcal{T}(12)$ is much simpler since the distinctions of positional relations can be reliably made without sophisticated measurements.

After player 1 has changed his direction of movement, five of the motion patterns change (see italic numbers in Table 11). The situation is completely different in that the white attacker now has no one to pass the ball to. However the motion pattern search algorithm is capable of telling apart these situations. The discussed scene ended by Wanchope (no. 1 in Fig. (7) kicking the ball into the net, changing the score to $2: 3$ for Costa Rica. This scene and several other scenes show that in fact $\mathcal{T} \mathcal{L} \mathcal{T}$ motion patterns and their combinations make significant distinctions regarding team behaviours, as can be comprehended by further typical scenarios which are presented in [3].

\subsection{Complex Spatiotemporal Patterns}

In this section we explain how to describe more complex situations by combining complex spatial with temporal patterns.

Definition 5 (Complex Spatiotemporal Motion Pattern). A complex spatiotemporal motion pattern is a conjunctive set of motion patterns of a number of $k>2$ objects for successive time intervals.

As an instance of a complex spatiotemporal problem, we exemplarily describe an offside situation which is defined by the Fédération Internationale de Football Association (FIFA) [4] as follows: A player is in an offside position if he is nearer to his opponents goal line than both the ball and the second last opponent. In addition to that there are some restrictions to the offside law. Thus, a player is not in an offside position if he is in his own half of the playfield or he is level with the second last opponent or he is level with the last two opponents. There is, furthermore, no offside offence if a player receives the ball directly from a goal kick or a throw-in or a corner kick which reduces the states we have to verify. Detecting offside situations we analyse motion patterns among $k \geq 4$ objects, at least two offensive players, an opponent and the ball. In general, to detect an offside position it is almost sufficient to regard two successive scenes. However, to identify an offside offence in which a striker is involved in active play or interfering with an opponent or gaining an advantage by being in an offside position, successive time intervals have to be taken into account. Fig. 10 shows 


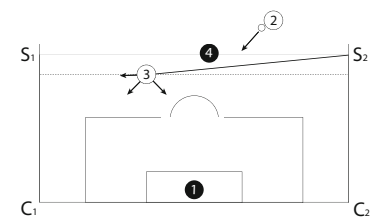

\begin{tabular}{llllll}
\hline & $S_{1}$ & $S_{2}$ & \multicolumn{2}{l}{$C_{2}$} & \multicolumn{2}{l}{$C_{1}$} \\
\hline$\leftarrow i$ & $21,23,24$ & $21,22,24$ & $22,23,24$ & $21,22,23$ \\
$i \rightarrow$ & $22,23,24$ & $21,22,23$ & $21,22,24$ & $21,23,24$ \\
$\leftarrow o$ & $21,22,23$ & $22,23,24$ & $22,23,24$ & $21,22,23$ \\
$o \rightarrow$ & $21,23,24$ & $21,22,24$ & $21,22,24$ & $21,23,24$ \\
\hline
\end{tabular}

Fig. 10. No. 2 passes the ball to his teammate no. 3 who holds an offside position (left); possible initial positions and directions for motion patterns (right)

a situation where a player no. 2 passes the ball to his teammate no. 3 who holds an offside position. Generally, the offside area can be defined by the orthogonal intersection outgoing from the second last opponent and the sidelines of the pitch. In this case, the offside area is limited by the vertices $S_{1}, S_{2}, C_{1}, C_{2}$. Thus, the challenge of identifying an offside position corresponds to the issue of determining the objects' position regarding a particular area. That is, we determine if a player is contained in the offside area $A$ or not by analysing motion patterns with respect to the vertices of $A$. Fig. 10 shows general distinctions which can be made in this context outgoing from possible positions (inside or outside) and possible moving directions (left or right). For example, a combination of motion patterns for player no. 3 and a time interval $[0,1]$ exists: $\left(3, S_{1}, 22,0,1\right),\left(3, S_{2}, 22,0,1\right)$, $\left(3, C_{2}, 21,0,1\right)$ and $\left(3, C_{1}, 21,0,1\right)$. In this case, we can unambiguously infer the initial position (inside) and direction (right) of the player.

\section{Summary}

In this paper we propose motion patterns as a qualitative representation to describe spatial and temporal behaviours in the soccer domain. It shows that these motion patterns can be directly projected onto symbolic patterns. That this works has been demonstrated for all 88 games of the simulation Championships in 2007. We also discuss temporal and spatial granules to draw up motion patterns among objects. We learn that, when being combined, these result motion patterns will enable much more complex scenarios and will form the basis in future work for plan recognition [8] by means of argumentation frameworks [2].

\section{References}

1. André, E., Binsted, K., Tanaka-Ishii, K., Luke, S., Herzog, G., Rist, T.: Three robocup simulation league commentator systems. AI Magazine 22(1), 57-66 (2000)

2. Bench-Capon, T.J.M., Dunne, P.E.: Argumentation in artificial intelligence. Artif. Intell. 171(10-15), 619-641 (2007)

3. Bode, G.: WM Analyse. Fussball training 24, 39-47 (2006)

4. Fédération Internationale de Football Association (FIFA). Laws of the game $2007 / 2008$ (2007)

5. Gottfried, B.: Representing short-term observations of moving objects by a simple visual language. Journal of Visual Languages and Computing (2007) 
6. Gottfried, B., Witte, J. (Sprado). Representing spatial activities by spatially contextualised motion patterns. In: Lakemeyer, G., Sklar, E., Sorrenti, D.G., Takahashi, T. (eds.) RoboCup 2006: Robot Soccer World Cup X. LNCS (LNAI), vol. 4434, pp. 330-337. Springer, Heidelberg (2007)

7. Hirano, S., Tsumoto, S.: Finding interesting pass patterns from soccer game records. In: Boulicaut, J.-F., Esposito, F., Giannotti, F., Pedreschi, D. (eds.) PKDD 2004. LNCS, vol. 3202, pp. 209-218. Springer, Heidelberg (2004)

8. Kautz, H., Allen, J.: Generalized plan recognition. In: AAAI 1986, pp. 32-37. AAAI Press, Menlo Park (1986)

9. Kiefer, P., Schlieder, C.: Exploring context-sensitivity in spatial intention recognition. In: Gottfried, B. (ed.) 1st Workshop on Behaviour Monitoring and Interpretation (BMI 2007), vol. 296, pp. 102-116. CEURS Proceedings (2007)

10. Laube, P., Imfeld, S., Weibel, R.: Discovering relative motion patterns in groups of moving point objects. International Journal of Geographical Information Science 19(6), 639-668 (2005)

11. Miene, A., Visser, U., Herzog, O.: Recognition and prediction of motion situations based on a qualitative motion description. In: Polani, D., Browning, B., Bonarini, A., Yoshida, K. (eds.) RoboCup 2003. LNCS (LNAI), vol. 3020, pp. 77-88. Springer, Heidelberg (2004)

12. Musto, A., Stein, K., Eisenkolb, A., Röfer, T., Brauer, W., Schill, K.: From motion observation to qualitative motion representation. In: Habel, C., Brauer, W., Freksa, C., Wender, K.F. (eds.) Spatial Cognition 2000. LNCS, vol. 1849, pp. 115-126. Springer, Heidelberg (2000)

13. Riedmiller, M., Gabel, T.: Brainstormers 2D - team description 2007. In: Visser, U., Ribeiro, F., Ohashi, T., Dellaert, F. (eds.) RoboCup 2007: Robot Soccer World Cup XI. LNCS (LNAI), vol. 5001. Springer, Heidelberg (2008)

14. Visser, U., Weland, H.: Using online learning to analyze the opponent's behavior. In: Kaminka, G.A., Lima, P.U., Rojas, R. (eds.) RoboCup 2002. LNCS, vol. 2752, pp. 78-93. Springer, Heidelberg (2003)

15. Zimmermann, K., Freksa, C.: Qualitative Spatial Reasoning Using Orientation, Distance, and Path Knowledge. Applied Intelligence 6, 49-58 (1996) 